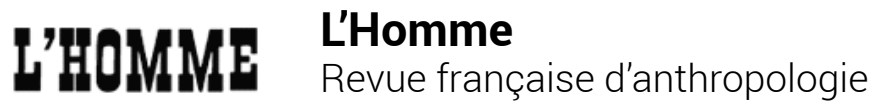

171-172| 2004

Musique et anthropologie

\section{Une anthropologie de la musique classique occidentale}

La culture comme "autre"

\section{Bruno Nettl}

\section{(2) OpenEdition \\ Journals}

\section{Édition électronique}

URL : http://journals.openedition.org/lhomme/24939

DOI : 10.4000//homme.24939

ISSN : 1953-8103

\section{Éditeur}

Éditions de l'EHESS

Édition imprimée

Date de publication : 1 décembre 2004

Pagination : 333-351

ISSN : 0439-4216

Référence électronique

Bruno Nettl, « Une anthropologie de la musique classique occidentale », L'Homme [En ligne], 171-172 I 2004, mis en ligne le 01 janvier 2006, consulté le 10 décembre 2020. URL : http://

journals.openedition.org//homme/24939; DOl : https://doi.org/10.4000/lhomme.24939 


\title{
Une anthropologie de la musique classique occidentale
}

\author{
La culture comme "autre"
}

Bruno Nettl

LES CHERCHEURS du domaine connu aujourd'hui sous le nom d'" ethnomusicologie " ont dû se battre pour se faire une place en relation avec un certain type d'"autre». Dans son article marquant de 1855, Alexander John Ellis (1885: 485), bien qu'employant l'expression "Various Nations» ("différentes nations ») au niveau du titre, expliquait qu'il avait dû laisser de côté cet autre: On the Musical Scales of All Nations ("Sur les gammes musicales de toutes les nations »), nous engageant ainsi sur la voie d'une inclusivité universelle. Mais pas pour longtemps. Au début du XX siècle, dans un article intitulé "Die Probleme der vergleichenden Musikwissenschaft ", Erich M. von Hornbostel souligne qu' "un domaine d'étude nouveau et spécialisé est confronté au besoin de justifier son existence ". Puis, alors qu'il affirme que les problèmes de ce domaine " amèneront directement à des questions plus générales, comme celles de l'origine et du développement de la musique et de la nature du beau en musique ", il propose des méthodes qui sont explicitement destinées à l'étude d'expressions musicales ne relevant pas du contexte de celles, savantes et populaires, qui ont cours en Occident. En 1930, Curt Sachs publie l'une des premières introductions sur le sujet, intitulée Vergleichende Musikwissenschaft: Musik der Fremdkulturen - la musique de l' "étranger " ou de l'"autre» - , et Robert Lachmann (1929: 1) fait ressentir le besoin d'élaborer une méthodologie spécifique que motive l'importance de la transmission orale dans ce type de musique. Si nous avons glissé de l'universel à l' " autre " - car cet autre partage une même forme de transmission -, les recherches des années 1950 mettent quant à elles l'accent sur l'étude de l'«autre» au simple motif qu'il se trouve "à l'extérieur». C'est ainsi que, en 1959, Jaap Kunst voue l'ethnomusicologie à l'étude de la musique traditionnelle du monde entier, incluant "l'acculturation musicale et le rôle joué par l'hybridation" (Kunst 1959: 1), mais exclut de ce domaine de recherche "les musiques savantes et populaires occidentales ». 
En fait, l'ethnomusicologie consiste encore très largement en l'étude de l' "autre » musical. Cependant, la méthodologie actuelle - avec ses avantages et ses inconvénients - est plus axée sur le caractère étranger du chercheur que sur l'«altérité » des cultures étudiées. De sorte que les travaux les plus récents des ethnomusicologues occidentaux tendent à considérer le monde du point de vue du chercheur et de son "altérité" personnelle.

Le lien entre étranger et autochtone a glissé d'une conscience interculturelle à une conscience intraculturelle, et les questions de différence - de genre, d'orientation sexuelle, de déviance sociale ou tout autre écart par rapport à un courant général donné - ont également trouvé une place au sein de l'ethnomusicologie. On remarquera que l'étude de la musique classique occidentale a fini par devenir une sous-division de ce domaine.

De toutes les musiques du monde, la musique savante occidentale est bien évidemment, et de loin, celle qui a donné lieu au plus grand nombre d'études, surtout de la part des musicologues se définissant comme historiens de la musique, mais aussi de celle des théoriciens et analystes, esthéticiens, philosophes, psychologues, enseignants et critiques. Que peuvent alors apporter les ethnomusicologues à cette somme de données et à toutes ces interprétations? La réponse est claire : un regard sur la musique savante occidentale à travers ses us et coutumes. Pour analyser ce regard, on pourrait être tenté de relancer le débat à propos des définitions de l'ethnomusicologie, mais que l'on me permette de court-circuiter la pédanterie qui menace ici : 1) en renvoyant le lecteur au long compendium d'Alan P. Merriam (1977), paru il y a vingt-cinq ans et toujours valable jusque sur ce point précis ; 2) en exposant la double définition que je donne aux profanes pour décrire mon domaine: l'approche comparative des musiques du monde entier, et l'approche anthropologique de chacune d'entre elles.

La tâche consiste à définir ici cette " approche anthropologique " pour laquelle je considère le concept de culture comme central en dépit des critiques dont ce dernier a fait l'objet. Il s'agit de la culture au sens défini par Edward B. Tylor (1871: 1), c'est-à-dire un ensemble de valeurs centrales et de principes de base déterminant la vie d'une société, et la façon dont celle-ci s'interprète elle-même et interprète son monde. L'anthropologie est l'étude des êtres humains, en particulier par le biais de leur culture, et l'étude anthropologique de la musique relie le champ de la musique à d'autres domaines culturels, en examinant comment le premier soutient, reflète ou même contredit les seconds.

Si, au sein de cette approche finalement peu éloignée de celle des historiens traditionnels, il existe un point sur lequel les ethnomusicologues - ces anthropologues de la musique - peuvent prétendre avoir apporté une contribution, c'est bien l'inclusion - l'inclusion de leur point de vue, de leur domaine culturel, et des sociétés mondiales (au sein de la plupart desquelles ils sont inévitablement des étrangers). La majorité des anthropologues se voient effectivement comme des étrangers par rapport à la société qu'ils étudient, et même lorsqu'ils affirment faire partie de celle-ci, ils conservent inévitablement ce caractère, puisque aucun «autochtone» de la société en question n’est engagé dans le type de travail qu'ils 
effectuent. Les anthropologues européens qui étudient les différents aspects de la culture occidentale sont donc eux aussi forcément des étrangers, et c'est en utilisant les méthodes qu'ils ont élaborées pour tenter d'appréhender les cultures extérieures à la leur qu'ils réussiront le mieux à appréhender celle-ci. Lorsque je me suis lancé dans l'étude de ma propre culture musicale, de façon très spécifique puisque je me suis focalisé sur les écoles de musique des Universités du Midwest américain -, j'ai cherché à poser le même type de questions que j'avais posées pour tenter de comprendre d'autres cultures (Nettl 1995 : 1-9).

Mais il existe aussi un autre aspect dans lequel la relation «autochtone/étrangers" joue un rôle dans l'étude par les ethnomusicologues de «leur" propre musique : l'ethnomusicologie elle-même a été - ou, plus significatif encore, s'est elle-même vue comme - une sorte d'étrangère parmi les sciences humaines. De façon un peu extrême, on peut dire qu'il s'agit d'une discipline consacrée à la musique, forme artistique qui se trouve être, sur de nombreux points, la "déviante" de la culture occidentale : celle que certains associent aux individus déviants de la société. Les ethnomusicologues se consacrent eux aussi souvent au type de musique qui dévie le plus de leurs propres normes culturelles puisqu'ils font fi de l'esthétique pure, préférant épouser le relativisme. L'étude de la musique classique occidentale constitue donc pour eux un acte quelque peu séditieux, tout comme l'ethnomusicologie dans son ensemble a des accents subversifs par rapport à la culture musicale occidentale.

\section{Les traditions académiques}

Une proportion non négligeable des ethnomusicologues sont issus des cursus anthropologiques et se considèrent d'ailleurs comme des anthropologues. Ce sont eux qui, à la base, ont défini les contours de l'ethnomusicologie en tant qu'étude de l'«extérieur". Mais qu'en est-il du rôle de la musique pour les anthropologues qui ne sont pas spécialistes de ce domaine et souhaitent malgré tout traiter les relations entre des secteurs de la culture, musique comprise, au sein de ce "tout complexe" ? Certaines des grandes figures de l'histoire de l'anthropologie ont bien sûr entretenu des affinités avec le domaine de la musique. Le nom qui vient en premier à l'esprit est celui de Claude Lévi-Strauss, qui a centré la préface d'un de ses travaux les plus connus, Le Cru et le cuit, sur la musique, et intitulé les parties et chapitres du livre en utilisant le nom donné à certaines formes et compositions de la musique savante occidentale (sonate, thème et variation, cantate, etc.). Ce n'est pas l'interprétation de la musique par ce grand maitre qui est en question ici, mais plutôt l'idée que, dans son ouvrage, le rapport est considéré comme constant entre les mythes des Indiens d'Amérique du Sud, qui exposent les principes fondateurs de leurs sociétés, et la musique dans ses manifestations spécifiquement occidentales.

Plus près de moi, au cours de mon travail sur les Blackfoot du Montana et de l'Alberta, j'ai bénéficié des conseils d'Oscar Lewis, auteur de travaux remarqués sur la "culture de la pauvreté » en Inde et en Amérique latine, et qui avait rédigé 
sa thèse de doctorat sur les Blackfoot (Lewis 1941, 1942). Oscar Lewis, lui-même très bon chanteur d'opéra, avait acquis une grande connaissance de multiples aspects de la culture blackfoot, sans toutefois parvenir à comprendre quel intérêt je pouvais trouver aux sons exotiques de la musique blackfoot. Siegfried F. Nadel, anthropologue britannique d'origine autrichienne, effectue lui aussi cette distinction entre la musique et le reste de la culture. En 1951, après avoir été chef d'orchestre et musicologue, et publié des travaux d'ethnomusicologie, il changea de direction, soutenant que les caractéristiques des styles musicaux ne sont pas liées à la structure des autres domaines culturels : "Il n'y a pas de lien entre l'organisation du groupe et [...] l'homophonie ou la polyphonie musicales [...]. En art, le style existe donc par lui-même, ne motivant ni ne présupposant aucune relation sociale déterminée " (Nadel $1951: 88$ ).

En prenant pour objet la musique, de nombreux anthropologues se détournent de la notion d'" autre", et adoptent le point de vue typique du musicien classique occidental selon lequel il est impossible de parler sérieusement de musique sans disposer de certains concepts et d'un certain vocabulaire. Peut-être pensent-ils également que l'approche de n'importe quelle musique, même celle de la société dans laquelle ils vivent, fournit un degré suffisant d' "altérité ». Quoi qu'il en soit, l'examen de manuels nord-américains d'introduction à l'anthropologie montre que la musique bénéficie de très peu d'attention - au contraire des arts visuels et de tradition orale - et que, lorsqu'il en est question, les illustrations sont généralement puisées dans la musique artistique occidentale ou dans le jazz (Nettl 2002)*.

Si cet article se propose d'envisager la musique classique occidentale d'un point de vue anthropologique, d'autres disciplines au sein des sciences sociales ont bien évidemment ouvert la voie et exercé des influences importantes sur le sujet. Je me contenterai de citer quelques figures majeures : Theodor W. Adorno (1962), dont les études comprennent une analyse des relations entre processus musicaux et sociaux, et des différentes fonctions sociales des musiques "sérieuse » et de « divertissement» ("E-musik» et "U-musik» en allemand) ; Pierre Bourdieu (1979) qui, entre autres choses, a analysé le rôle de la musique classique dans les goûts musicaux de différentes strates de la société française ; Kurt Blaukopf (1982), dont l'une des contributions importantes est l'interprétation du rôle des médias dans les changements survenus au sein de la musique occidentale ; Elias Canetti (1960), écrivain et essayiste, qui a porté un regard attentif sur le chef d'orchestre en tant que figure politique indésirable; Jacques Attali (1977), pour son analogie entre activité musicale et économie politique.

Ces exemples montrent assez clairement comment, dans la tradition universitaire européenne dont l'étiquette "sociologique" provient peut-être d'un souci pratique de généralisation, le rôle de la culture musicale contemporaine au sein de la société occidentale a été envisagé bien avant l'entrée en scène des ethno-

* La situation est en tous points comparable au sein de l'université française : voir le début de l'argument de Bernard Lortat-Jacob \& Miriam Rovsing Olsen publié en tête de ce numéro de L'Homme (pp. 7 sq. et notes). Ndlr. 
musicologues. Les intellectuels mentionnés plus haut, dont les intérêts musicaux provenaient largement d'un environnement et d'une expérience classiques, ont eu beaucoup d'influence sur de jeunes chercheurs qui étudièrent la culture musicale artistique, notamment dans le monde anglo-saxon, lesquels se donnèrent le nom d' "ethnomusicologues ». Ce qui distingue ces derniers du groupe de théoriciens européens cités plus haut, c'est leur expérience de l'étude des cultures et systèmes musicaux "étrangers", expressément non-occidentaux, ainsi que les questions fondamentales qu'au sein de la leur ils essayent de poser.

On peut donc caractériser des domaines tels que l'ethnomusicologie par les problèmes fondamentaux que celle-ci entend explorer et qu'elle tente de résoudre. Mais les ethnomusicologues ont-ils formulé de telles questions si l'on considère l'ensemble de la littérature? En 1905, Erich M. von Hornbostel se contenta de mentionner l'"origine et le développement de la musique» (Hornbostel, ibid.), et je ne crois pas qu'Alan P. Merriam eût jamais énoncé la définition de son domaine sous la forme d'une simple question; il voyait dans l'ethnomusicologie une étude de la musique dans la culture (et plus tard la pensa "en tant que" culture), la culture au sens où l'avait défini Edward B. Tylor (1871), à savoir l'interprétation de la musique dans ses relations aux autres domaines. Pour établir les choses précisément, Alan P. Merriam élabora, en guise de guide, un modèle en trois parties toutes liées entre elles: idées, comportements, sons (Merriam 1964). Cette formulation met en jeu les champs fondamentaux de la musique, et leur interrelation est centrale. Je me souviens d'ailleurs d'une conversation au cours de laquelle il avait explicitement soutenu que la "relation des domaines entre eux " était également centrale pour toute anthropologie. J'ai, pour ma part, essayé d'identifier quelle était, à mes yeux, l'interrogation cruciale de notre domaine, et $j$ 'ai abouti à la formulation suivante: "Qu'est-ce qui détermine le style musical qu'une société décide d'adopter?» (Nettl 1983 : 234-240). C'est la première question qui me soit venue à l'esprit lorsque, étudiant, portant peu d'intérêt aux " universaux " mais fasciné par l'immense diversité des musiques du monde, je pensai que notre travail consistait à les expliquer. Ce souci semble aussi être à la base de l'étude d'Alan Lomax, conventionnellement nommée "cantométrique", qui a d'ailleurs fourni une réponse provisoire bien connue: "le chant favori d'une culture reflète et renforce le type de comportement essentiel à son effort principal de survie et à ses institutions sociales centrales et dirigeantes" (Lomax et al. 1968: 133). John Blacking (1973:26) a apporté une réponse similaire : «il y a forcément un rapport entre les structures de l'organisation humaine et les structures des sons, rapport découlant des interactions humaines ". Moins impressionné par la diversité que par les universaux, John Blacking se demandait donc, dès le titre de son ouvrage, How Musical is Man? - suggérant par là que tous les humains possèdent un minimum substantiel de musicalité qu'il nous revient d'explorer. Wolgang Suppan (1984: 26-28), quant à lui, émet trois propositions de recherche: la musique comme comportement humain (plutôt qu'ensemble de productions), comme partie de l'univers symbolique humain, et comme outil. Timothy Rice 
(1987: 473) a lui aussi donné sa version de la question: «comment les gens construisent-ils historiquement la musique, comment l'entretiennent-ils socialement, comment la créent-ils et la vivent-ils individuellement ?». On constate que la tradition tripartite se perpétue; mais Timothy Rice propose également, plus simplement, "comment les gens font-ils de la musique?» Une question simple en apparence, mais derrière laquelle se cachent de nombreuses hypothèses et interrogations fondamentales.

On notera qu'aucune de ces propositions n'établit de distinction entre la musique savante occidentale et celle du reste du monde. Les anthropologues ont en principe toujours intégré la première à la seconde dans leurs ouvrages et leurs formulations de fond. Pourquoi ont-ils attendu tout ce temps pour l'observer de façon plus directe?

\section{La musique comme totalité}

Il existe sûrement plusieurs raisons à cela. L'une d'entre elles, au moins, mérite d'être retenue : la complexité du système culturel occidental. On peut certes se demander si les cultures tribales ou villageoises sont réellement plus simples (même si elles sont démographiquement modestes), mais c'est en tout cas ainsi que les musicologues et les scientifiques semblaient voir la chose. Leur préférence pour ce qu'il considéraient comme des cultures "plus simples" n'était pas tant motivée, à mon avis, par une croyance en un caractère de primauté ou de primitivité que par l'idée que l'anthropologie est l'étude d'une totalité culturelle. Du milieu du XXe siècle jusqu'à aujourd'hui, le désir des ethnomusicologues est de comprendre les systèmes musicaux - tous les aspects de la musique et de la vie musicale dans la société - dans leur globalité. Cette entreprise a rarement été couronnée de succès, mais le titre de multiples ouvrages et articles montre clairement que c'était bien là l'objectif.

Il est indéniable qu'une société, même petite, est un organisme extrêmement complexe, ce qui a mené les ethnomusicologues vers deux directions aux dénominations peut-être choisies par souci pratique : l'approche macroscopique (ou macrocosmique) et l'approche microscopique (ou microcosmique). La première tente d'atteindre l'exhaustivité en relevant tous les détails sur de larges horizons; la seconde sélectionne un aspect de la culture musicale se prêtant particulièrement, peut-être par effet de convergence et reflet, à la compréhension de son essence, en reproduisant dans un microcosme les principes du tout. Les chercheurs qui ont essayé de mener à bien l'approche exhaustive, dite "macroscopique" - celle-ci requérant une organisation ingénieuse et l'analyse d'une immense quantité de données - ont été peu nombreux et n'ont rencontré qu'une audience limitée. Parmi eux, le plus marquant demeure Alan P. Merriam qui, dans son ouvrage Ethnomusicology of the Flathead Indians (1967), propose un modèle pour cette approche, sa trilogie inachevée, An African World (1974), devant constituer une étude encore plus complète et contextualisée de la musique basongye d'Afrique centrale. De même, La Vie musicale d'un village, de 
Constantin Brailoiu (1960), tente de prendre en compte, individuellement et en groupe, tous les chants et tous les chanteurs d'une même culture. L'hypothèse selon laquelle la culture musicale n'est véritablement comprise que dans la musique "traditionnelle" pose un problème commun à tous ces cas; Alan P. Merriam, par exemple, a ignoré la connaissance et l'usage qu'avaient la population flathead des musiques populaire, religieuse, scolaire et classique.

À l'inverse, l'approche microcosmique a été privilégiée dans de nombreuses études. Parmi celles à retenir, signalons Sound and Sentiment, de Steven Feld (1982), qui prend comme point de départ la taxinomie des sons chez les Kaluli ainsi qu'un mythe essentiel, un peu à la manière de Claude Lévi-Strauss; et Why Suyá Sing, d'Anthony Seeger (1988), qui échafaude l'image de toute la culture musicale à partir d'une observation détaillée de la «cérémonie de la souris ». Ces études, ainsi que d'autres, nous montrent que, même pour des sociétés de cent cinquante individus, il est difficile de cerner l'ensemble d'une culture musicale.

L'application de ces approches à la musique classique occidentale (qui, tout comme celle des Flathead, évolue au sein d'un contexte) est décourageante, mais à partir des années 1970, de nouvelles études virent le jour, qui avaient bénéficié de tous ces travaux préparatoires. Voici quelques points de repères: The Hidden Musicians, de Ruth Finnegan (1989), est très voisine de l'approche «macrocosmique " adoptée par Alan P. Merriam dans son travail sur les Flathead. Mais cette étude globale s'attarde sur ce qui provient de la tradition classique dans une petite ville anglaise (musique de concert professionnelle, travaux amateurs et scolaires, création de musique informelle); elle differe de celle d'Alan P. Merriam en ce qu'elle tient compte de l'ensemble de l'expérience musicale de la communauté. Son statut mérité d'important travail ethnomusicologique provient en partie de ce qu'elle entremêle les activités musicales de toutes sortes au-delà des interprétations formelles. Le livre de Henry Kingsbury (1988), Music, Talent and Performance, définit la culture de la musique classique occidentale par l'observation d'un petit conservatoire de l'Est des États-Unis considéré comme une institution centrale par le fait qu'elle focalise tout sur le talent, valeur érigée en élément déterminant qui régit les activités à tous les niveaux. L'idée qu'il existe un concept prépondérant spécifié par la culture qui définit une culture musicale particulière, semble contredire la croyance ethnomusicologique en une comparabilité générale, mais elle pourrait trouver un parallèle dans les études sur la musique du Moyen-Orient, comme celles d'Hiromi Lorraine Sakata (1983) et de John Baily (1988). Celles-ci expliquent clairement que l'ambivalence islamique intégriste vis-à-vis de la musique est centrale pour la compréhension de la vie musicale dans sa globalité. L'étude réalisée par Philip Bohlman (1989) sur la culture musicale des locuteurs de langue allemande et tchèque immigrés en Isräl s’appuie sur la spécificité du répertoire musical pour comprendre et définir l'intégration de cette communauté. À l'instar de recherches sur des traditions très variées comme, par exemple, le travail d'Helen Myers (1998) sur une communauté indienne de Trinidad ou encore celui de Margaret Sarkissian (2000) sur une communauté d'origine portugaise en Malaisie, Philip Bohlman montre un groupe qui, dans sa vie quotidienne, est totalement intégré dans la 
société israélienne et marque son origine commune par une adhésion à la tradition de Bach, Mozart et Schubert (qui ne jouaient pourtant pas un rôle de marqueurs dans leur pays d'origine), principalement par le biais de concerts de musique de chambre privés. S'inspirant d'Alan P. Merriam, Philip Bohlman n'examine pas les activités musicales (celles de la musique populaire, par exemple) auxquelles les membres de la société sont susceptibles de prendre part dans d'autres contextes, mais observe les salles, les méthodes et les institutions qui dérivent d'un répertoire classique.

Les recherches que nous venons d'évoquer cernent leur sujet à partir d'une société, d'un groupe de gens circonscrit, dont les auteurs examinent la musique qui, pour eux, est centrale. À l'inverse, Kay Shelemay (2001), dans son étude sur le «mouvement de musique ancienne" de Boston, observe ce qu'on pourrait décrire comme une activité impliquant très peu d'individus de la sphère déjà spécialisée de la "musique classique ". S’ils se consacrent également à cette dernière, les interprètes et les amateurs de "musique ancienne " (antérieure au XVII siècle) forment une société musicale à part entière, dont le nombre de membres se chiffrent par dizaines d'individus plutôt que par centaines. À l'inverse de l'étude de Philip Bohlman d'une société aux origines ethniques communes qui choisit un répertoire musical comme emblème, Kay Shelemay présente une société dont l'existence découle de son adhésion à un répertoire musical spécifique.

Ces études effectuées par des chercheurs qui se considèrent comme des ethnomusicologues se distinguent par le fait qu'elles s'appuient sur des travaux concernant des musiques non occidentales, ou en tout cas, des recherches menées sur l'"autre».

\section{Les Écoles de musique. Étude de cas dans le Midwest américain}

Je souhaite à présent faire part de quelques observations concernant la culture musicale au sein de laquelle j'ai vécu pendant plus de cinquante ans - celle des Écoles de musique universitaires du Midwest - en résumant et révisant certains de mes travaux antérieurs (Nettl 1992, 1989a) et en me focalisant sur différentes appoches structurelles qui seront éclairées par le systèmes politique, économique et religieux. La perspective adoptée est définie plus haut sous l'appellation « microcosmique », fondée donc sur l'idée que les structures du domaine individualisé et ses subdivisions reflètent celles du tout; j'ai également opéré un lien entre mes remarques et la littérature sur l'«autre». Enfin, je me suis concentré sur les manières dont les groupes, les individus et les métaphores musicales sont liés entre eux en termes de pouvoir, d'influence et de contrôle.

Sur plusieurs points, par comparaison avec des départements universitaires typiques, les Écoles de musique constituent des institutions autoritaires. Si, au cours des séances de travaux pratiques, les étudiants ont le droit d'être en désaccord avec l'enseignant, ils ne contestent pas leur professeur de violon, ni le chef d'orchestre ni, par extension, le directeur ou le doyen. Mais plus encore que les 
maîtres en place, ce sont les grands compositeurs qui dirigent l'École, ces compositeurs dont les noms gravés sur les bâtiments ou dans les salles de concert évoquent des valeurs et des principes inaltérables. Ces hommes sont présentés au public de l'École comme une sorte de panthéon, ou de cour royale, avec sa mythologie, ses textes sacrés, son sacerdoce et ses concepts analogues à ceux de Satan et du péché.

\section{La structure des mythes}

À l'instar de la fonction du mythe kaluli rapporté par Steven Feld à propos du "garçon qui était devenu un oiseau ", il existe des mythes qui exposent les valeurs fondamentales de nos Écoles de musique et expliquent, tout comme les «vrais » mythes, notre comportement dans le présent et dans le passé, même s'il ont parfois peu à voir avec la version historique des événements. Ils expliquent nos valeurs donc, mais ne les déterminent pas et, tout comme l'attitude de notre société envers les pratiques et répertoires musicaux, ces mythes sont susceptibles de changer. Dans ma tentative d'interprétation du rapport entre musique et culture chez les Blackfoot, j'ai recherché un mythe central et choisi celui où une femme mariée suit dans l'eau l'homme-castor mythique qui l'a séduite. Elle en ressort quatre jours plus tard avec un enfant dont elle prend soin avec son mari. L'homme-castor rend visite à ce dernier qui, en grand chasseur, possède des peaux de chaque animal de la nature dans sa tente, et lui révèle, un à un, les chants permettant de contrôler le pouvoir surnaturel des animaux et des oiseaux. Après chaque chant, interprété une seule fois, le chasseur donne à l'homme-castor la peau de l'animal chanté. C'est ainsi que les Blackfoot acquirent l'humanité, la musique et la "cérémonie du castor ", celle-ci dotée d'une grande importance à leurs yeux. Cette histoire explique quantité de choses par rapport à la musique : son origine et son pouvoir surnaturels, le reflet du système culturel dans le système musical, ou encore l'idée que ce sont les animaux qui enseignent les chants, au cours de visions et lors d'une seule écoute (Nettl 1992, 1989a : 129-130).

À la recherche de parallèles dans la musique classique occidentale et les Écoles de musique, j’ai repensé à la période pendant laquelle j’avais été étudiant. À l'époque, on était confronté à plusieurs récits explicatifs faisant référence aux hiérarchies et à notre tendance à regarder le monde en termes de dualités et de triades. La mythologie gravitait autour de compositeurs faisant office d'unités de base de la pensée musicale (à la différence, peut-être, des œuvres ou des types d'interprétation) comme, par exemple, Bach en super-intellectuel et figure tutélaire du monde de la musique savante tel que nous le connaissons. Les trinités avaient leur importance : les classiques viennois (Haydn-Mozart-Beethoven), les trois B (Bach-Beethoven-Brahms), la seconde école viennoise (Schoenberg-BergWebern). Le monde de la musique était aussi l'expression de dualités - dérivées de l'opposition majeur/mineur - présentées sous la forme de paires de compositeurs: Léonin/Pérotin dans le Paris du Moyen-Âge, Ockeghem/Obrecht, Palestrina/di Lasso, Cesti/Cavalli et d'autres, jusqu'au XVIII' siècle; Bach/Haendel, 
Haydn/Mozart, Beethoven/Schubert, Schubert/Schuman, Chopin/Lizst, Brahms/ Wagner, Smetana/Dvorak, et bien d'autres encore, chaque paire présentant d'importants points communs mais également des éléments de contradiction. La paire Bach/Haendel était ainsi brandie pour symboliser le lien entre modulation et sens de la mise en scène; Brahms/Wagner, entre conservatisme et révolte; Beethoven/ Schubert, dont les différents types de musicalité avaient été interprétés par Sir George Grove (Gramit 1993) selon les concepts masculin/féminin, incarnant l'opposition entre intellect et sentiment; invention harmonique, formelle et invention mélodique; et finalement force, pouvoir versus grâce. La musique de Schubert était forcément pourvue de toutes les qualités possibles. En fait, c'est apparemment le souci de présentation au monde et aux étudiants des corrélations entre les différentes qualités et valeurs de la musique qui a donné lieu à la création de mythes dont les personnages étaient les compositeurs - solution la plus pratique.

Cette mythologie comprenait également deux visions différentes de l'histoire, assénées en des termes très simples : 1) La musique avait progressé jusqu'à l'époque de Mozart et Beethoven puis, malgré tous les efforts déployés, avait entamé un déclin irrésistible en dépit de sursauts occasionnels, ne nous laissant d'autre possibilité que de contempler les cimes inaccessibles des grands classiques. 2) Le point le plus important concernant l'histoire de la musique est le changement constant de style. L'innovation constitue ipso facto une amélioration, mais le prix à payer est une difficulté croissante du public à saisir la musique - d'où l'idée que, plus la musique est de qualité, moins le public à même de l'apprécier est nombreux.

\section{Le panthéon}

Dans les Écoles, le répertoire, l'interprétation, la pratique et les répétitions, ainsi que l'histoire de la musique sont très largement traités par rapport aux compositeurs, ceux-ci étant présentés selon une hiérarchie rappelant les anciens panthéons européens et en tête de laquelle se trouvent presque invariablement Bach, Beethoven et Mozart, mais également Haydn, Haendel, Schubert, Wagner, et parfois même des maitres de musique "ancienne" tels Palestrina; on y repère rarement un nom postérieur à Brahms ou qui ne soit pas allemand. Les étudiants, les enseignants, le public parlent tous de ces compositeurs en termes révérencieux, et plus le rang du compositeur est élevé dans la hiérarchie, moins la critique à son endroit est tolérée. Se plaindre que tel morceau de Beethoven est ennuyeux fait courir le risque d'être traité d'hérétique, et on dit communément d'un compositeur «mineur » qu'« il ne vaut pas Mozart».

Sur les plaques commémoratives, dans les publications comme dans les programmes, les compositeurs - tous des hommes - apparaissent comme une sorte de famille. Mozart et Beethoven, présentant à la fois continuité et différences, sont en haut de l'affiche: ils sont les plus joués, les plus à l'abri de la critique et les plus explicitement mythologisés. Bach a sa place, bien sûr, mais son rôle est plutôt celui d'une sorte de père des divinités les plus vigoureuses, un genre de Zeus ou de 
Wotan constituant un repère absolu (chacun doit apprendre ses « 48 » et son style d'harmonie) et qui s'introduit dans les affaires de tous (il est impératif d'éviter, comme lui, les quintes parallèles, et quelle que soit la qualité avant-gardiste du travail effectué, il est indispensable de composer une fugue pour démontrer son sérieux). Le couple fondamental est suivie de figures légèrement en retrait Schubert, Haydn, Haendel, Wagner -, et la perception d'un caractère musical masculin/féminin, comme celui évoqué dans le cas de Beethoven et Schubert, ajoute à la notion de famille. Certains compositeurs, reconnus comme faisant partie du cercle des « grands " - tels Wagner et, éventuellement, Lizst - sans toutefois parfaitement intégrer le cadre, remplissent la fonction de scélérats. Dans toute cette structure, la mythologie entretient des liens, parfois seulement ténus, avec l'histoire. Ainsi Wagner peut-il être méprisé pour certains aspect de sa vie, tandis que le caractère de sa musique reste, quant à lui, toujours soumis à interprétation.

Le mythe fondamental de la musique classique occidentale, historiquement fragile et dont la valeur est à rechercher sans doute du côté du rôle de l'art, me semble être celui de la paire Mozart/Beethoven. Cette observation amène à prendre en compe la situation de la classe dirigeante. Mozart et Beethoven sont considérés comme les deux plus grands compositeurs et leur fonction dans la pédagogie générale est de représenter le lien entre facteurs humains et surnaturels dans l'art. Alors que les historiens et les analystes sérieux pourraient tout à fait décrire ces deux hommes en des termes similaires - prodigieusement doués, bourreaux de travail, créateurs d'une musique extrêmement complexe et résolvant des problèmes essentiels -, Mozart et Beethoven sont présentés de manière fort différente aux étudiants et au public profane.

Mozart, doué d'une inspiration surnaturelle, composait à une vitesse étonnante, sans effort, et ses phrases musicales ont des manières d'évidence dans leurs enchaînements. Enfant prodige, miraculeux, doué d'une capacité de mémoire et d'improvisation époustouflantes, son talent ne fut toutefois guère apprécié de son vivant et il mourut précocement, de façon tragique; sa mort fut annoncée par le commanditaire de son Requiem et marquée par une atmosphère particulièrement ténébreuse. Tout au long de son existence, il conserva à peu près le même style musical. Ses œuvres furent le fruit d'une sorte de pouvoir surnaturel, d'où la grande attention portée à sa mort mystérieuse. Mais surtout, Mozart était un génie-né, concept européen anciennement associé aux idées d'immobilité sociale et d'élites. Dans les salles de classe, Mozart est le premier musicien dont on parle aux enfants, puisqu'on le considère comme le plus abordable, qui a composé dès son plus jeune âge et n’a jamais vraiment perdu son esprit juvénile.

Dans cette description de la pédagogie conventionnelle, le pendant de Mozart est Beethoven. Personnalité différente, difficile d'accès, à l'image de sa musique. L'opposé de Mozart sur bien des aspects. Si la mort de Mozart est auréolée de mystère, c'est la naissance de Beethoven qui reste énigmatique, concernant la date autant que l'origine familiale. Beethoven avait une allure sombre et soucieuse, vécut de terribles souffrances et de fréquentes déceptions, sans oublier sa tragique surdité. Sa musique ne lui venait pas facilement, il est évident que l'écri- 
ture lui demandait un travail acharné; l'écoute de son œuvre exige beaucoup d'efforts et convient mieux aux personnes d'un certain âge. Son style a évolué tout au long de sa vie, si bien que ses dernières œuvres sont extrêmement différentes des premières. Il n'eut pas d'enfants, mais un neveu qu'il avait adoré le déçut profondément. Considéré comme un rebelle luttant pour se libérer de toutes sortes de chaînes, handicapé par sa surdité qui domine l'image que nous avons de lui, il traversa une existence difficile, travaillant d'arrache-pied, polissant ses œuvres des années durant avant de les achever. Prenant ses responsabilités d'artiste très au sérieux, il sacrifia de nombreuses choses au profit de la spiritualité de sa musique. Génie au même titre que Mozart, il dut toutefois galérer pour le devenir (Nettl 1989a). Mozart est connu pour son extravagance - comme l'illustrent sa correspondance et ses textes obscènes, par exemple -, tandis que Beethoven passe pour être d'un sérieux imperturbable, ce qui est historiquement faux. La facilité avec laquelle la musique de Mozart donne l'impression d'avoir été composée se reflète dans son association avec la douceur, jusque dans les produits utilisant le nom du compositeur, comme le chocolat Mozartkuglen, les liqueurs, les innombrables «Café Mozart » et autres boutiques dans le monde entier. Associé au nom de Beethoven, ce compositeur laborieux, je n'ai rien découvert d'autre qu'un restaurant et une société de déménagement de pianos, malgré la grande légèreté dont cet homme fit incontestablement preuve - dans sa vie comme dans son œuvre (Kinderman 1995 : 255-258).

Les valeurs importantes de la musique classique occidentale - inspiration et travail, cohérence et évolution, sérieux et sens de l'humour - sont donc présentées aux étudiants et aux profanes par le biais des différences légendaires (occasionnellement véridiques) entre ces deux grands compositeurs et leur musique. Il est intéressant de noter que, aux État-Unis, les concerts proposent très fréquemment des œuvres de Mozart et de Beethoven, mais celles-ci sont rarement jouées le même soir.

\section{Organisation sociale}

Les relations entre groupes de gens dans la vie musicale relèvent à la fois des règles fondamentales de la société et de celles du système musical. En Iran, au cours des années 1960, j'ai eu l'occasion de faire les constatations suivantes à propos de la culture de la musique classique et de ses pratiquants: les musiciens, essentiellement des solistes évitant de jouer à plusieurs, se réunissaient au sein de petites écoles pratiquant un même style - plus petites et plus nombreuses que les gharanas de musique indienne décrites par Daniel Neuman (1980 : 145-167) -, reflétant ainsi l'importance de l'individualisme fréquemment soulignée par les Perses et ceux qui les étudient. Associée à la valeur conférée à l'imprévisibilité du comportement, la musique improvisée et non métrique (rythmiquement imprévisible) jouissait d'un plus grand prestige que la musique composée et métrique. En écho à l'ambivalence de l'islam shiite, les interprètes de cette dernière n'aimaient pas se voir désignés comme professionnels, préférant l'idée d'un amateurisme érudit. Le statut peu élevé de ce type de musique profita à un nombre 
disproportionné de musiciens non musulmans ou issus des minorités, et une terminologie fut élaborée qui ne qualifiait pas de "musique " la musique vocale, à textes, improvisée et non métrique, qui, accoutisquement, se rapproche au plus près de la lecture du Coran (Baily 1988 : 146-153, Ibsen al-Faruqi 1985).

Les relations sociales au sein des Écoles de musique d'Amérique du Nord reflètent à la fois celles de la société en général et celles du système musical. Ce qui en ressort est une société dans laquelle tous les gens sont égaux en théorie mais où, en fait, chacun se livre à une lutte permanente pour le pouvoir entre les classes et les différents types de groupes sociaux. Voici quelques observations:

Tout d'abord, il existe une opposition et une compétition pour occuper un rôle hégémonique entre certaines paires : interprètes contre chercheurs et compositeurs; musiciens contre enseignants; chanteurs contre instrumentistes; etc. Ensuite, il y a une compétition en triangle entre étudiants, enseignants et administratifs - acolytes officieux du directeur -, ces trois groupes étant aisément identifiables par leur type de vêtements. Ainsi étudiants et enseignants font-ils alliance pour demander plus de moyens, d'espace et de flexibilité dans les programmes, tandis qu'administratifs et enseignants font de même pour exiger plus de matières pour les étudiants et leur faire passer plus de temps au sein des ensembles dans lesquels ils doivent jouer - orchestres, groupes, chœurs. Enfin, étudiants et administratifs unissent leurs forces pour pousser les professeurs à se consacrer plus intensivement à leur enseignement et à réduire le temps passé à donner des concerts et des cours en dehors de l'École. La constatation générale est que les jeux d'alliance sont incessants.

Troisième point, déjà évoqué plus haut, les Écoles de musique des universités américaines se conforment moins au style relativement démocratique de l'institution que les départements d'histoire ou d'anthropologie, par exemple, et affichent plus de formes autoritaires de direction, une chose généralement acceptée par l'ensemble des individus concernés. Le directeur ou doyen, souvent riche d'une expérience préalable d'"administrateur", et rarement nommé parmi les professeurs locaux, tend à disposer de pouvoirs presque dictatoriaux, à rester en fonction pendant une longue période et, lorsqu'il quitte son poste (ou est démis de ses fonctions), à partir enseigner ailleurs que dans l'institution qu'il a dirigée. Ce parcours est analogue à celui du chef d'orchestre type et c'est d'ailleurs au sein de l'orchestre - ou d'autres ensembles, comme les chœurs (Adorno 1962: 104118) - qu'on trouve l'expression du modèle social le plus clair. La position particulière du chef d'ensemble se distingue aisément par son habillement, son entrée, sa relation à l'auditoire - à noter que le directeur de l'École partage certains de ces attributs. Avant le début d'un concert, tel un contremaître, le maître de concert présente l'orchestre, une fois celui-ci "accordé", au chef d'orchestre, figure du grand propriétaire. Le directeur de l'École se lance parfois dans un discours aux relents esclavagistes, parlant de "mon professeur de hautbois " et de "mon directeur d'opéra ". Les hommes nommés à ce poste font corps grâce à d'importantes organisations professionnelles, frayant plus entre eux que les administrateurs d'autres disciplines universitaires. Mais la société hiérarchique 
existe aussi dans la musique elle-même, dans l'orchestre, avec le chef d'orchestre, le maitre de concert, les chefs de section et le placement numéroté jusqu'au concept de solo et d'accompagnement, qu'illustre parfaitement le piano, pour lequel la plupart des morceaux privilégient les parties de main droite par rapport aux accompagnements de main gauche.

Quatrièmement, l'École est une hiérarchie de répertoires et de personnes qui leur sont étroitement associés. Il s'agit d'une institution dont les composantes sont reliées entre elles en un ensemble de cercles concentriques. Bien que cela ne soit pas formellement admis, l'objectif fondamental de l'École - hormis le projet très large de tout enseigner de toute la musique - est la formation d'interprètes de musique classique d'Europe occidentale, au centre de laquelle se trouvent ses éléments les plus paradigmatiques, l'orchestre symphonique et le piano. Les musiques, "nouvelle " et " ancienne " (" médiévale», de la Renaissance et du début de l'ère baroque), complètent le répertoire classique (essentiellement des œuvres composées entre 1700 et 1920). Le cercle suivant engloberait des fanfares - y compris celles qui défilent en uniforme lors des événements sportifs -, jouant des marches, de la musique légère et des arrangements. Plus loin se trouveraient le jazz, puis la musique non occidentale, comme les gamelans, la sanza africaine et les ensembles de percussions, ou encore les musiques «folkloriques » d'Europe de l'Est. Sur le cercle le plus éloigné du centre seraient placés différents types de musique populaire - musique country, blues, rock - qui ne sont que rarement intégrés à l'appareil de l'École.

Deux remarques à propos de cette structure :

$1^{\circ}$ La taxinomie des musiques est renforcée par les costumes typiques des interprètes (une convention qui s'est défaite autour de 2001) : la musique "classique» est jouée par des hommes en smoking et des femmes en robes souvent longues et noires tout aussi protocolaires. Pour les concerts de musique «nouvelle », on adopte les pulls à col roulé, et le costume d'époque est de rigueur pour la musique "ancienne». Les interprètes de musique non occidentale, quant à eux, portent des vêtements plus ou moins traditionnels, suivant l'origine de la musique, tandis que les groupes de jazz universitaires se parent d'habits colorés. Les conventions concernant la musique populaire veulent que chacun des membres de l'ensemble soit vêtu différemment. Par ailleurs, certains ensembles d'importance secondaire essaient parfois, semble-t-il, de se rapprocher du centre de l'attention en jouant habillés en tenue de soirée.

$2^{\circ}$ Les Écoles de musique universitaires américaines sont passées, dans les cinquante dernières années, d'une exclusivité pour la musique classique à une plus grande variété, ajoutant des répertoires et des musiques à leur programme selon une pratique plus ou moins colonialiste. Bien souvent, les nouveaux venus entrent par la porte de la musicologie avant d'intégrer peu à peu l'appareil officiel qui, somme toute, constitue l'essence de l'institution. Ainsi, dans les années 1950, la musique " ancienne » fut-elle d'abord enseignée dans les cours d'histoire sans faire beaucoup de détails, avant de glisser peu à peu vers la sphère des "collegia musica». Les cours de musiques africaine et asiatique étaient assurés 
par des ethnomusicologues avant que ces musiques ne fussent enseignées à des ensembles. Les gamelans, présentés comme des "travaux pratiques » dans le cursus d'ethnologie, devinrent des formations permanentes en-dehors du cadre scolaire et finirent par bénéficier d'un statut comparable à celui des orchestres européens. La musique de variété, quant à elle, existe dans la plupart des Écoles américaines comme sujet d'étude théorique et historique, mais n'est pas pratiquée. Lorsque des musiques n'appartenant pas aux premiers cercles entrent dans la sphère de l'interprétation, elles sont jouées dans les mêmes conditions que la musique classique, qui donc occupe la première place. Peu importe leur contexte d'origine, elles sont présentées lors de concerts de quatre-vingt dix minutes avec entracte, et l'auditoire se comporte comme s'il assistait à une représentation de musique symphonique : tout est fait pour juger de la valeur de la musique non centrale à partir du contexte de la musique centrale.

\section{Le répertoire en tant que société}

Des anthropologues tels que A. R. Radcliffe-Brown et S. F. Nadel considéraient le centre d'une totalité culturelle comme une organisation et une structure sociales, comme un système de relations entre les gens au sein de la société. Si l'on poursuit cette ligne de pensée, il paraît dès lors logique de mettre en relation les composantes de la musique et celles de la société. C'est ce qu'a fait Alan Lomax (1968), par exemple, qui soutenait que les différents types d'ensembles instrumentaux et vocaux sont déterminés par la nature des relations entretenues. Je suggère, quant à moi, que la musique peut également constituer une métaphore de la société. Ainsi, dans la musique savante de l'Inde du Nord, les principales unités de la pensée musicale sont classées en ragas, raginis et putras (ragas masculins, ragas féminins et enfantins). Le système de musique classique iranien peut être interprété par les musiciens comme étant composé de douze modes regroupés selon une organisation rappelant le modèle familial : shur ("mère des dastgahs") et ses quatre dastgahs secondaires ou " enfants", chahargah et segah (en partie analogues) tenant le rôle de "frères ", etc. L'opposition entre l'idée que les douze dastgahs sont tous égaux et l'idée qu'ils entretiennent des relations distinctes et sont d'importance variable reflète celle qui existe entre la notion d'égalité des êtres devant l'islam et les rapports hiérarchiques de la société iranienne. Les liens entre les instruments d'un gamelan sont eux aussi fréquemment expliqués par rapport à la société de cour javanaise traditionnelle (Hood 1971 : 244-245).

Dans la musique classique occidentale, la terminologie des structures sociales est présente dans les "familles " d'instruments ; les "familles » musicales jouent elles aussi un rôle majeur dans l'histoire de la musique - ainsi en est-il de l'accent généralement porté sur celles de Bach, Mozart et Liszt ou Wagner. Mais je pousse plus loin l'analogie en suggérant que les membres de la culture de la musique classique occidentale, et plus particulièrement le public des Écoles de musique universitaires américaines, peuvent considérer que les répertoires musicaux et les œuvres entretiennent entre eux des relations analogues à celles des membres de la société. Selon 
cette idée, il apparaît que le répertoire musical incarne un type de société particulièrement inégalitaire. L'importance - c'est-à-dire le pouvoir - provient du poids, c'est-à-dire de la puissance sonore, de la longueur, de la complexité : les ouvres majeures sont toutes de grandes symphonies, des messes et requiems, et, par-dessus tout, des opéras. Peu de compositeurs peuvent espérer atteindre le statut de "grand maître " s'il ne s'essaie à ces trois super-genres. Les concerts et les récitals sont généralement structurés selon une hiérarchie plaçant les œuvres maîtresses au plus près de l'entracte (avant ou après), une structure qui reflète l'idée de «plat de résistance». La "classe dirigeante" de la musique, et en un certain sens la plus populaire, est composée des genres qui affichent les plus nombreux degrés d'autorité, à savoir l'opéra et le concerto. Néanmoins, le concept d'une sorte de «sacerdoce» musical, symbole du sérieux et de certaines valeurs éternelles, typiquement associé à un public d'un certain âge ainsi qu'à une pratique religieuse réelle, s'incarne en une musique dont les composantes sont davantage mises sur un pied d'égalité, comme par exemple les quartuors à cordes, les fugues ou les œuvres fuguées, dans lesquelles trois à cinq "voix " jouissent d'un même statut, délaissant le principe du solo-accompagnement mis en œuvre dans la plupart des musiques.

S'il est envisageable de considérer les remarques établies dans ce travail comme base de discussion à propos de la musique classique au sein de la culture occidentale dans son ensemble, je ne souhaite toutefois pas les étendre au-delà de la sphère des Écoles de musique universitaires du Midwest des États-Unis. Il y a peu de chances que l'étude des Écoles de musique fournisse des données ethnographiques fondamentalement nouvelles, comme cela a peut-être été le cas pour les recherches sur les Suyá ou les Blackfoot; c'est seulement par les suggestions de mises en relation et d'interprétation que nous pouvons espérer apporter une contribution à la discipline. Au lecteur de juger si un point de vue anthropologique permet de mieux comprendre le rapport entre la musique classique occidentale et sa culture et sa société, et si l'étude de la musique de l'«autre" nous aide à comprendre notre propre culture. Si le lecteur réagit en disant : «Soit, mais il n’y a là rien de nouveau ", alors notre contribution aura été bien mince. Mais s'il déclare: "Je sais tout cela depuis longtemps, mais je n'y avais jamais pensé de cette façon, en considérant mon monde musical dans le contexte d'un monde de musiques différentes ", cela laisse espérer que l'anthropologie de la musique a son mot à dire à ceux qui étudient la culture musicale artistique contemporaine.

\section{Traduit de l'anglais (États-Unis) par Manuel Benguigui.}

MOTS CLÉS/KEYWORDS: ethnomusicologie/ethnomusicology - concept de culture/culture concept - institutions - éducation/education - mythe/myth. 
Adorno, Theodor W.

1962 Einleitung in the Musiksoziologie.

Frankfurt, Suhrkamp.

Al-Faruqi, Lois Ibsen

1985 "Music, Musicians, and Muslim

Law ", Asian Music, $17:$ 3-36.

Attali, Jacques

1977 Bruits : essai sur l'economie politique de la musique. Paris, Presses universitaires de France.

Baily, John

1988 Music of Afghanistan: Professional Musicians in the City of Herat. Cambridge, Cambridge University Press.

Blacking, John

1973 How Musical Is Man? Seattle, University of Washington Press.

\section{Blaukopf, Kurt}

1982 Musik im Wandel der Gesellschaft.

Munich, Piper.

\section{Bohlman, Philip Vilas}

1989 The Land Where Two Streams Flow: Music in the German-Jewish Community of Israel. Urbana, University of Illinois Press.

\section{Bourdieu, Pierre}

1979 La Distinction : critique sociale du jugement. Paris, Éditions de Minuit.

\section{Brailoiu, Constantin}

1960 La Vie musical d'un village : recherches sur le repertoire de Dragus (Roumanie) 19291932. Paris, Institut universitaire roumain Charles Ir.

\section{Canetti, Elias}

1960 Masse und Macht. Hambur, Suhrkamp. (trad. franç. : Paris, Gallimard, 1966).
Ellis, Alexander J.

1885 "On the Musical Scales of Various Nations ", Journal of the Society of Arts 33 (no. 1688) : 485-527.

Feld, Steven

1982 Sound and Sentiment: Birds, Weeping, Poetics, and Song in Kaluli Expression. Philadelphia, University of Pennsylvania Press.

Finnegan, Ruth

1989 The Hidden Musicians: MusicMaking in an English Town. Cambridge, Cambridge University Press.

Gramit, David

1993 "Constructing a Victorian Schubert : Music, Biography, and Cultural Values", Nineteenth-Century Music 17 : 65-78.

\section{Grove, Sir George}

1889 Grove's Dictionary of Music and Musicians. London, Macmillan.

Hood, Mantle

1971 The Ethnomusicologist. New York, McGraw-Hill.

Hornbostel, Erich M. von

1905-6 «Die Probleme der vergleichenden Musikwissenschaft ", Zeitschrift der internationalen Musikgesellschaft 7 : 85-97.

\section{Kinderman, William}

1995 Beethoven. Berkeley, University of California Press.

\section{Kingsbury, Henry}

1988 Music, Talent, and Performance: a Conservatory Cultural System. Philadelphia : Temple University Press. 
Kunst, Japp

1959 Ethnomusicology. The Hague, Nijhoff.

1960 «Indonesia ", The Columbia World

Library of Folk and Primitive Music, vol. 8. KL-210.

Lachmann, Robert

1929 Die Musik der aussereuropäischen

Natur- und Kulturvölker.Wildpark-Potsdam,

Athenaion. ("Handbuch der

Musikwissenschaft»)

Lévi-Strauss, Claude

1964 Le Cru et le cuit. Paris, Plon.

\section{Lewis, Oscar}

1941 "Manly-Hearted Women Among the

North Piegan ", American Anthropologist 43 : 173-87.

1942 The Effects of White Contact Upon

Blackfoot Culture. New York, American

Ethnological Society,

Lomax,. Alan et al.

1968 Folk Song Style and Culture.

Washington, American Association for the

Advancement of Science.

\section{Merriam, Alan P.}

1964 The Anthropology of Music. Evanston, Northwestern University Press.

1967 Ethnomusicology of the Flathead Indians. Chicago, Aldine Publication.

1967 Ethnomusicology of the Flathead Indians. Chicago, Adline Pub. Co.

1974 An African World: the Basongye Village of Lupupa Ngye. Bloomington, Indiana University Press.

1977 "Definitions of "Comparative Musicology" and "Ethnomusicology": An Historical-Theoretical Perspective", Ethnomusicology 21 : 189-204.

Myers, Helen

1998 Music of Hindu Trinidad: Songs from the Indian Diaspora. Chicago, University of Chicago Press.
Nadel, Siegfried F.

1951 The Foundations of Social

Anthropology. London, Cohen and West.

Nettl, Bruno

1983 The Study of Ethnomusicology. Urbana, University of Illinois Press.

1989a «Mozart and the

Ethnomusicological Study of Western

Culture ", Yearbook for Traditional Music

$21: 1-16$.

1989b Blackfoot Musical Thought:

Comparative Perspectives. Kent, Kent State University Press.

1992 «Heartland Excursions : Exercises in Musical Ethnography ", World of Music 34 (1) : 8-34.

1995 Heartland Excursion. Urbana, University of Illinois Press.

2002 Encounters in Ethnomusicology, a Memoir. Warren, Harmonie Park Press.

Neuman, Daniel M.

1980 The Life of Music in North India. Detroit, Wayne State University Press.

Rice, Timothy

1987 «Toward the Remodeling of

Ethnomusicology ", Ethnomusicology 31 : 469-88.

Sachs, Curt

1930 Vergleichende Musikwissenschaft: Musik der Fremdkulturen. Heidelberg, Quelle und Meyer.

\section{Sakata, Hiromi Lorraine}

1983 Music in the Mind: The Concepts of Music and Musicians in Afghanistan. Kent, Kent State University Press, 1983

\section{Sarkissian, Margaret}

2000 D'Albuquerque's Children: Performing Tradition in Malaysia's Portuguese Settlement. Chicago, University of Chicago Press. 
Seeger, Anthony

1988 Why Suyá Sing? Cambridge,

Cambridge University Press.

Shelemay, Kay Kaufman

2001 "Toward an Ethnomusicology of the Early Music Movement ", Ethnomusicology 45: 1-29.
Suppan,Wolfgang

1984 Der musizierende Mensch :

Eine Anthropologie der Musik. Mainz,

Schott.

Tylor, Edward B.

1871 Primitive Culture. London, J. Murray.

RÉSUMÉ/ABSTRACT

Bruno Nettl, Une anthropologie de la musique classique occidentale. - L'ethnomusicologie, autrefois exclusivement consacrée à l'étude des traditions musicales transmises oralement, a développé, au cours des deux dernières décennies, un intérêt croissant pour la musique artistique occidentale, en particulier dans ses manifestations contemporaines. Cet essai se focalise essentiellement sur les problèmes méthodologiques que soulève la présentation d'un panorama complet de cette culture musicale complexe, en l'abordant dans le contexte de l'histoire de l'ethnomusicologie et en proposant une étude de la vie musicale des Écoles de musique universitaires (conservatoires) du Midwest américain. Les approches sont les suivantes: 1) la culture musicale en tant que mythe; 2) les "grands" compositeurs en tant que panthéon; 3) l'organisation sociale des enseignants et des étudiants; 4) le répertoire musical en tant que métaphore de la société et de ses interaction et organisation coutumières.
Bruno Nettl, The Anthropology of Western Art Music: My Culture as the "Other". — The field of ethnomusicology, once exclusively devoted to the study of orally transmitted musical traditions, has in the last two decades begun to approach the contemplation of the Western art music tradition, particularly in its contemporary manifestation. This essay is concerned principally with methodological problems of organizing a comprehensive panorama of this complex musical culture, seeing it in the context of the history of ethnomusicology and providing as a case study the musical life of university schools of music (conservatoires) in the Midwestern U.S.A. The approaches are 1) the study of musical culture as myth ; 2) the interpretation of the "great" composers as a pantheon; 3) the social organization of teachers and students; and 4) a view of the musical repertory as a metaphor of a society with customary organization and interaction. 